\title{
TTILE
}

\section{Cobalt thin films prepared by chemical vapor deposition from cobaltous acetate}

$\operatorname{AUTHOR}(S)$ :

MARUYAMA, T; NAKAI, T

\section{CITATION:}

MARUYAMA, T ...[et al]. Cobalt thin films prepared by chemical vapor deposition from cobaltous acetate. APPLIED PHYSICS LETTERS 1991, 59(12): 1433-1434

\section{ISSUE DATE:}

1991-09-16

URL:

http://hdl.handle.net/2433/43523

\section{RIGHT:}

Copyright 1991 American Institute of Physics. This article may be downloaded for personal use only. Any other use requires prior permission of the author and the American Institute of Physics. 


\title{
Cobalt thin films prepared by chemical vapor deposition from cobaltous acetate
}

\author{
Toshiro Maruyama and Tsuyoshi Nakai \\ Department of Chemical Engineering, Faculty of Engineering, Kyoto University, Kyoto 606, Japan
}

(Received 1 May 1991; accepted for publication 20 June 1991)

\begin{abstract}
Cobalt thin films were prepared by a low-temperature atmospheric-pressure chemical vapor deposition method. The raw material was cobaltous acetate which is nontoxic and easy to handle. At a reaction temperature of $300^{\circ} \mathrm{C}$, [111]-oriented polycrystalline films can be obtained on amorphous substrates. Increasing the $\mathrm{H}_{2}$ partial pressure over the requirement for both the highest deposition rate and lowest resistivity promotes the crystallization with [111] preferential orientation of the film without affecting the crystallite size. Cobaltous acetate appears to offer a viable alternative to cobalt acetylacetonate for low-temperature cobalt film production.
\end{abstract}

Cobalt thin films are attractive in their application to the memory elements. The cobalt layers have traditionally been prepared by vacuum deposition and electrolytic method in liquid phase. An alternative is to deposit cobalt by chemical vapor deposition (CVD) methods: ${ }^{1}$ the pyrolysis of cobaltous acetylacetonate or cobalt nitrosyl tricarbonyl at $400-450^{\circ} \mathrm{C}$; the thermal decomposition or hydrogen reduction of $\mathrm{CoI}_{2}$ vapor at a temperature higher than 800 or $600{ }^{\circ} \mathrm{C}$; the pyrolysis of cobalt dicyclopentadienyl at $400-450{ }^{\circ} \mathrm{C}$; or hydrogen reduction $\left(\right.$ at $400-450{ }^{\circ} \mathrm{C}$ ) of $\mathrm{CoCl}_{3}$ vaporized at $60-150{ }^{\circ} \mathrm{C}$. A relatively low-temperature $\left(325-350^{\circ} \mathrm{C}\right)$ process is hydrogen reduction of cobaltous acetylacetonate. ${ }^{2}$

In this letter, cobaltous acetate, which is nontoxic and readily available, is proposed as a source material in the low-temperature atmospheric-pressure CVD method. The films were deposited by hydrogen reduction of cobaltous acetate on various flow rates of hydrogen gas, and effects of $\mathrm{H}_{2}$ flow rate on deposition rate, resistivity, and crystallinity of the film are discussed.

Cobaltous acetate tetrahydrate $\left(\left(\mathrm{CH}_{3} \mathrm{COO}\right)_{2} \mathrm{Co}\right.$. $4 \mathrm{H}_{2} \mathrm{O}$ ) of reagent grade was used as the source material. It is easier to obtain and cheaper than cobaltous acetylacetonate. Figure 1 shows a schematic representation of the experimental setup. Cobaltous acetate was heated at a temperature of $300^{\circ} \mathrm{C}$ and the generated gas was entrained by nitrogen carrier gas. The flow rate of the carrier gas was $300 \mathrm{~cm}^{3} / \mathrm{min}$. Hydrogen gas was injected into the nozzle and mixed with the source gas. The flow rate of hydrogen gas was ranged from 300 to $1000 \mathrm{~cm}^{3} / \mathrm{min}$.

A $76 \times 26 \mathrm{~mm}$ borosilicate glass plate was used as the substrate. The substrate was placed in the closed-tube reactor heated by an external electric furnace. The substrate temperature was $300^{\circ} \mathrm{C}$. The total pressure in the reactor was an atmospheric pressure.

The composition of the film was measured by $x$-ray photoelectron spectroscopy. The crystallinity of the film was analyzed by the $\mathrm{x}$-ray diffraction method with $\mathrm{Cu} K \alpha$ radiation. The electric resistivity was measured by the van der Pauw method.

Shiny smooth cobalt films were formed homogeneously on the borosilicate glass substrates at the reaction temperature of $300{ }^{\circ} \mathrm{C}$. The films were grown by the hydrogen reduction of cobaltous acetate. In an inert (nitrogen) atmosphere, no film was obtained. The films were highly adherent and showed no apparent peeling on glass substrates.

Figure 2 shows the deposition rate of cobalt film as a function of $\mathrm{H}_{2}$ flow rate. The deposition rate increases with increasing $\mathrm{H}_{2}$ flow rate from 300 to $600 \mathrm{~cm}^{3} / \mathrm{min}$. The highest deposition rate is obtained at a $\mathrm{H}_{2}$ flow rate above $600 \mathrm{~cm}^{3} / \mathrm{min}$. Figure 3 shows the resistivity of cobalt film as a function of $\mathrm{H}_{2}$ flow rate. The resistivity decreases with increasing $\mathrm{H}_{2}$ flow rate from 300 to $600 \mathrm{~cm}^{3} / \mathrm{min}$ and above $600 \mathrm{~cm}^{3} / \mathrm{min}$ it shows the lowest value which is close to the resistivity for bulk cobalt $\left(6.24 \times 10^{-6} \Omega \mathrm{cm}\right.$ at $20^{\circ} \mathrm{C}$ ). Thus, the partial pressure of hydrogen gas in its lower values affects the resistivity and the deposition rate. When the $\mathrm{H}_{2}$ flow rate is over $600 \mathrm{~cm}^{3} / \mathrm{min}$, the deposition rate and the resistivity each show constant value independent of the $\mathrm{H}_{2}$ partial pressure.

Figures $4(a)-4(d)$ show the $x$-ray diffraction patterns of the $\sim 100$-nm-thick cobalt films deposited on the borosilicate glass substrates. All patterns show peaks at $2 \theta=44.1^{\circ}$. The peak was admitted in the $\mathrm{x}$-ray diffraction pattern at the $\mathrm{H}_{2}$ flow rate larger than $600 \mathrm{~cm}^{3} / \mathrm{min}$. That is, the cobalt film is composed of crystallite with a predominant cubic structure with [111] preferential orientation. Furthermore, Fig. 4 shows that the intensity of the Co (111) peak increases with increasing flow rate of $\mathrm{H}_{2}$ gas. The full width at half maximum of the diffraction peak (111) yielded the crystallite size of $\sim 17 \mathrm{~nm}$, which is independent of $\mathrm{H}_{2}$ flow rate. Thus, an extra $\mathrm{H}_{2}$ partial

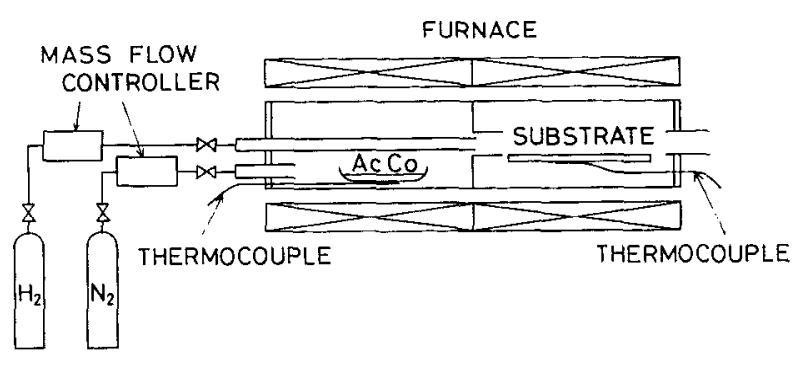

FIG. 1. Schematic representation of experimental equipment. 


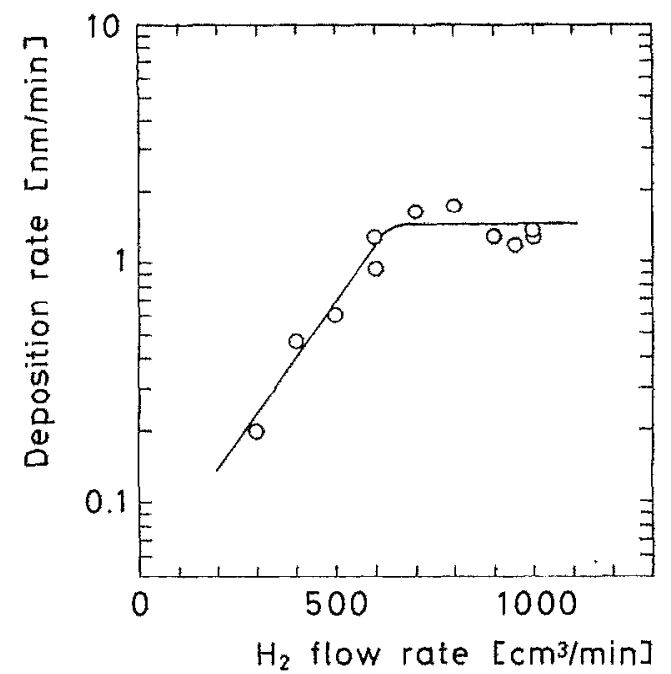

FIG. 2. Deposition rate of cobalt film as a function of $\mathrm{H}_{2}$ flow rate.

pressure over the requirement for both the highest deposition rate and lowest resistivity promotes the crystallization with [111] preferential orientation of the film without affecting the crystallite size.

In conclusion, cobalt thin films were prepared by a low-temperature atomspheric-pressure chemical vapor deposition method. The raw material was cobaltous acetate which is nontoxic and easy to handle. Polycrystalline films were obtained at a reaction temperature of $300^{\circ} \mathrm{C}$. Increasing the $\mathrm{H}_{2}$ partial pressure over the requirement for both the highest deposition rate and lowest resistivity promotes

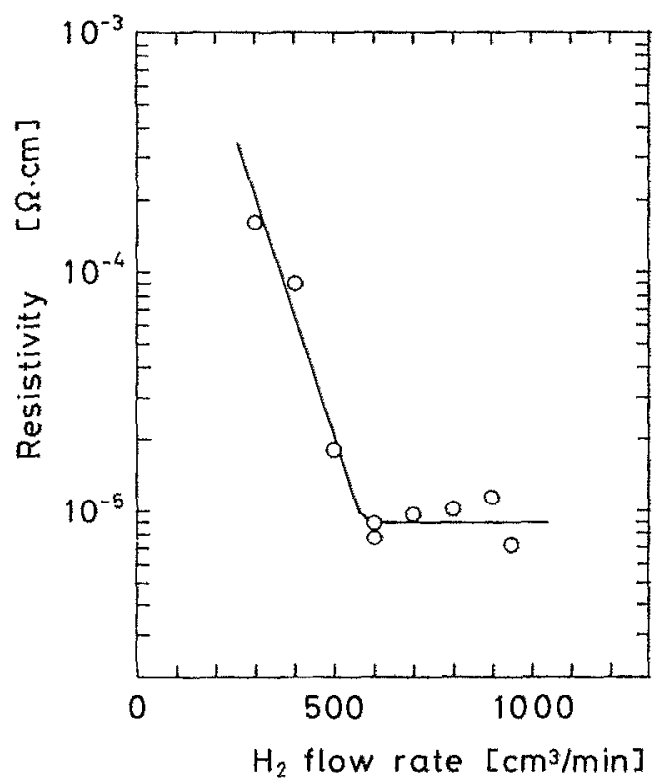

FIG. 3. Resistivity of cobalt film as a function of $\mathrm{H}_{2}$ flow rate.
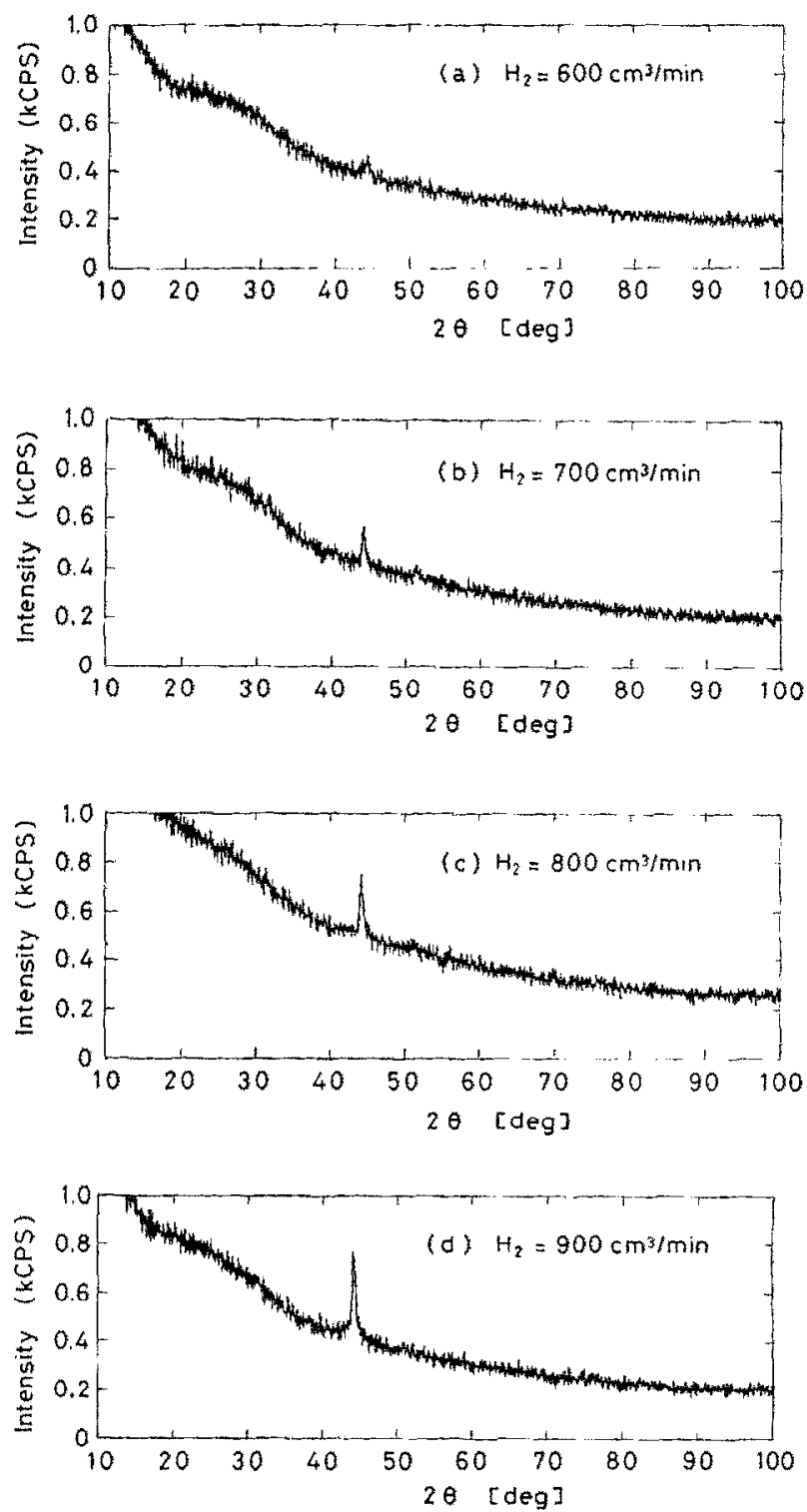

FIG. 4. X-ray diffraction pattern of $\sim 100$-nm-thick cobalt film for $\mathrm{H}_{2}$ flow rate: (a) $600 \mathrm{~cm}^{3} / \mathrm{min}$, (b) $700 \mathrm{~cm}^{3} / \mathrm{min}$, (c) $800 \mathrm{~cm}^{3} / \mathrm{min}$, and (d) $900 \mathrm{~cm}^{3} / \mathrm{min}$.

the crystallization with [111] preferential orientation of the film without affecting the crystallite size. Cobaltous acetate appears to offer a viable alternative to cobaltous acetylacetonate for low-temperature cobalt film production.

This work was supported by the Japan Securities Scholarship Foundation, Nissan Science Foundation, Iketani Science and Technology Foundation, Miyashita Foundation, Yazaki Science and Technology Foundation, and Nippon Sheet Glass Foundation.

'C. F, Powell, J. H. Oxley, and J. M. Blochre, Jr., eds., Vapour Deposition (Wiley, New York, 1966).

${ }^{2}$ E. J. Jablonowski, Cobalt 14, $28(1962)$. 